\title{
Anatomical Variations of the Circle of Willis in Males and Females on 3D MR Angiograms
}

\author{
Kawther A. Hafez, Nahla M. Afifi, Fardous Z. Saudi \\ Anatomy department, Faculty of Medicine, Ain Shams University
}

\begin{abstract}
Objective: The objective of the present work was to study the anatomical variations of the circle of Willis as regard its component vessels and their average diameters in a sample of adult Egyptians and to detect any sex-related differences in these variations.

Material and Methods: One hundred and twenty adult patients were observed (60 males and 60 females). They all had problems unrelated to any ischemic or vascular diseases, so they were considered as healthy control, concerning the morphology of the circle of Willis. In addition, ten cadavers' brains were obtained from the Anatomy department, Faculty of Medicine Ain Shams University for examination of the circle of Willis and for detection of any variations.

Results: The anatomical variations of the anterior part, posterior part and completeness of the circle were inspected. Also, the diameters of all component vessels were assessed.

The results indicated that, the anterior part of the circle was completed in $70 \%$ males and $75 \%$ females of the study sample. No statistically significant difference was detected between sexes. The most common variant of the anterior part was the single anterior communicating artery followed by the hypoplastic or absent anterior communicating artery.

The posterior part of the circle was completed in $48 \%$ males and $58 \%$ females. The most common variant was the bilateral posterior communicating arteries, followed by the unilateral posterior communicating artery. An entirely complete circle was found only in $45 \%$ of the entire population; and it was higher in the females than the in males. The vessels diameters were smaller in the females than in the males, except for the diameter of the posterior communicating artery. Cadavers' examination revealed six cases with complete circle, 3 cases of unilateral fetal posterior communicating and one case of absent posterior communicating artery.

Conclusion: The present study showed the amazing great variability of the anatomy of the circle of Willis in asymptomatic persons. Nevertheless, there were no marked differences between both sexes in most of the components and the mean diameters of the circle. Therefore, these anatomical variations have to be considered during radiological interpretation and would be reported in the current anatomy text to be aware of all these normal variations.
\end{abstract}

\section{Introduction}

Circle of Willis is located in the interpeduncular fossa and joins the two internal carotid arteries with the basilar artery to ensure continued blood supply to vital nervous tissues in cases of occluded cerebral arteries (William et al., 1995; Ray et al., 2007 ), (Fig.1). The proximal segment of the anterior cerebral artery can provide hemispheric cerebral blood flow from the contralateral internal carotid if the anterior communicating artery is patent and the basilar artery can supply collateral flow to the anterior circulation through the proximal segment of the posterior cerebral artery with reversal flow through the posterior communicating artery (Schomer et al., 1994). Ability of the circle of Willis to redistribute blood flow depends on its component vessels (Miralles et al., 1995). Patients who suffered from occluded internal carotid artery (ICA) but their circle of Willis with efficient collateral circulation have a risk of strokes than those without such collaterals (Hoksbergen et al., 2003, Eftekhar et al., 2006). Vessels of the circle of Willis vary in caliber, being often 
partially developed, sometimes even absent and about $60 \%$ of circles display anomalies (William et al., 1995). Higher prevalence of circle of Willis anomalies were detected in brains with signs of ischemic infarction (Hoksbergen et al., 2003). Identifying the functional collaterals of the circle of Willis assists in detection of patients with ICA stenosis at lower risk of strokes and transient ischemic attacks (Hederson et al., 2000; Hoksbergen et al., 2003). Knowledge of the collateral ability of the circle of Willis is important for neurosurgeons, vascular surgeons, and interventional radiologists when a procedure in the cerebral arteries is to be attempted (Hoksbergen et al., 2003). Magnetic resonance angiography is a non-invasive sensitive technique for detecting the anatomy of the circle of Willis (Stock et al., 1996). Three-dimensional time of flight magnetic resonance angiography (3D MRA) can provide valuable information regarding the collateral anatomy and function of the circle of Willis (Hoksbergen et al., 2003). Sex-linked variation in the circle of Willis was a point of controversy some authors proved statistically significant difference between the frequency of variations and sex (Horikoshi et al., 2002), but others excluded the relation between sex and variations (Macchi et al., 1996; Hartkamp et al., 1998 ). Accordingly, it was objective of the present work to study the anatomical variations of the circle of Willis as regard its component vessels and their average diameters in a sample of adult Egyptians and to detect any sex-related differences in these variations.

\section{Material And Methods}

\section{Material}

One hundred and twenty MR angiograms of adult patients (60 males and 60 females) of ages ranged between $40-70$ years (mean of 55 years) were obtained from the MRI unit, Ain Shams specialized hospital and Cairo centre for radiology. They all had problems unrelated to ischemic or vascular disease. Therefore, they were considered as healthy control as regard the anatomy of the circle of Willis. Patients were not included in the study if they had a medical history suggesting possible ischemic disease (stroke, transient ischemic attacks and dementia). Moreover, patients were excluded if they had history of diabetes mellitus, hypertension, any vascular or heart disease. A written consent was taken from all patients for the research.

In addition, ten cadaveric brains were obtained from the Anatomy Department Faculty of Medicine, Ain Shams University for examination of the circle of Willis and for detection of any variations.

\section{Methods (Miralles et al., 1995 ; Hederson et al., 2000; Merkkola et al., 2006,):}

The patients were subjected to threedimensional time of flight MR angiography of the circle of Willis by using 1.5 MR system. Imaging Parameters were 40/ 6.5; TR/ TE (time of repetition / time of echo), $20^{\circ}$ flip angle, $200 \times 150 \mathrm{~mm}$ field of view for the circle area, 225x 512 matrix, $0.67 \mathrm{x}$ $0.39 \mathrm{~mm}$ pixel resolution, three signals acquired, $1.4 \mathrm{~mm}$ section thickness. The 3D time of flight MR angiographic sequence required 4 minutes 30 seconds.

All MR angiograms were assessed on a special workstation, by using maximum intensity projections as well as source images. All the component vessels of the circle of Willis were assessed by measuring the diameter on the individual transverse 3D time of flight sections. Vessels that showed at least $1 \mathrm{~mm}$ in diameter were considered as hypoplastic.

Classification of variations of the circle of Willis (Overbeeke et al.,1991):

1-Anatomical variations in the anterior part of the circle of Willis (Fig 2)

The variants are:

a- Single anterior communicating artery; the ICA bifurcates into anterior and middle cerebral arteries.

b- Two (or more) anterior communicating arteries.

c- Medial artery of the corpus callosum arises from the anterior communicating artery. 
d- Fusion of the anterior cerebral arteries over a short distance.

e- Anterior cerebral arteries form a common trunk and split distally into two postcommunicating segments.

f- The middle cerebral artery (MCA) originates from the internal carotid artery (ICA) as two separate trunks.

g- Hypoplasia or absence of the anterior communicating artery.

h- One precommunicating segment of an anterior cerebral artery is hypoplastic or absent.

i- Hypoplasia or absence of an ICA, the contralateral precommunicating segment of the anterior cerebral artery gives rise to both postcommunicating segments.

J- Hypoplasia or absence of an anterior communicating artery and the MCA arises as two separate trunks.

\section{2-Anatomical variations in the posterior part of the circle of Willis (Fig 3)}

The variants are:

a- Bilateral posterior communicating arteries present.

b- Posterior cerebral artery originates predominantly from the ICA. This variant is known as a unilateral embryonic or fetal type posterior cerebral artery.

c- Bilateral embryonic (fetal) type posterior cerebral arteries.

d- Unilateral posterior communicating artery present.

e- Hypoplasia or absence of both posterior communicating arteries.

f- Unilateral fetal type posterior cerebral artery and hypoplasia or absence of the precommunicating segment of the posterior cerebral artery (P1).

g- Unilateral embryonic (fetal) type posterior cerebral artery and hypoplasia or absence of the contralateral posterior communicating artery.

h- Unilateral embryonic (fetal) type posterior cerebral artery and hypoplasia or absence of both a precommunicating segment (P1) of the posterior cerebral artery and the posterior communicating artery. i- Bilateral embryonic (fetal) type posterior cerebral arteries with hypoplasia or absence of both precommunicating segments. of the posterior cerebral arteries (P1).

j- Bilateral embryonic (fetal) type posterior cerebral arteries, with hypoplasia or absence of one precommunicating segment of a posterior cerebral artery.

\section{3-Anatomical variations of the whole circle of Willis}

A-The circle as a whole was considered complete when all vessel segments of both the anterior and the posterior part of the circle were visible and continuous (Fig.1) and demonstrated diameters of at least $1 \mathrm{~mm}$.

B- The circle was considered incomplete when both anterior and posterior vessels demonstrated a hypoplastic or absent segment.

C- The partially complete circles demonstrated either anterior or posterior hypoplastic or absent segment.

\section{Diameters of the vessels}

Average diameter measurements for each of the component vessels were measured for both sexes and the hypoplastic vessels were excluded from mean diameter calculations.

\section{Statistical analysis:}

All measures were averaged, mean and standard deviations were analyzed using student's $t$-test. P values $\leq 0.05$ was considered significant.

\section{Results}

\section{1-Anatomical variations in the anterior part of the circle of Willis (Table 1)}

The most common variation of the anterior part of the circle of Willis was the presence of two anterior cerebral arteries with single anterior communicating artery. It was 58.3\% in males and 55\% in females. (Table1:a, Fig.4). Two anterior communicating arteries were present in $11.7 \%$ males and $11.7 \%$ females (Table1,b, Fig.5). 
Medial artery of the corpus callosum that arises from the anterior communicating artery was not found in the present study (Table1,c). Fusion of the anterior cerebral arteries over a short distance was found in $5 \%$ males and $3.3 \%$ females (Table1,d, Fig.6). Anterior cerebral arteries formed a common trunk and split distally into two postcommunicating segments was found in $3.3 \%$ in males and 5\% in females (Table1,e, Fig.7).

Middle cerebral artery (MCA) originates from the internal carotid artery (ICA) as two separate trunks was not found in this study (Table1,f). Hypoplasia or absence of the anterior communicating artery was detected in $15 \%$ males and $20 \%$ females; so this was the next common variation after type a variation (Table1,g, Figs.8,9). Single precommunicating segment of an anterior cerebral artery was hypoplastic or absent detected in $6.7 \%$ males and 5\% females (Table1,h, Fig.6). Hypoplasia or absence of an ICA, the contralateral precommunicating segment of the anterior cerebral artery gives rise to both postcommunicating segments of the ICA was not detected in this work (Table1,i). Hypoplasia or absence of an anterior communicating artery and the MCA arises as two separate trunks was also not detected (Table1,j).

Therefore, males were more likely to have variations in the anterior part of the circle than the females, though this was not clinically significant.

\section{2-Anatomical variations in the posterior part of the circle of Willis (Table 2)}

The commonest variation of the posterior part of the circle was the presence of bilateral posterior communicating arteries. It was found in 30\% males and $26.6 \%$ females (Table 2, a Fig.4). Posterior cerebral artery originates predominantly from the ICA, which is known as a unilateral embryonic (fetal) type posterior cerebral artery was detected in $16.7 \%$ males and $20 \%$ females (Table2,b). Bilateral embryonic (fetal) type posterior cerebral arteries were found in $1.7 \%$ males and $8.3 \%$ females (Table 2,c Fig.7). Unilateral posterior communicating artery was present in $21.7 \%$ males and $20 \%$ females (Table 2,d) so it was the next common variation in the posterior part. Hypoplasia or absence of both posterior communicating arteries was detected in $13.3 \%$ males and $11.7 \%$ females (Table 2,e Figs. 8,9). Unilateral fetal type posterior cerebral artery and hypoplasia or absence of the precommunicating segment of the posterior cerebral artery were detected in 3.3\% males and 1.7 females (Table 2,f). Unilateral fetal type posterior cerebral artery and hypoplasia or absence of the contralateral posterior communicating artery were noticed in $6.7 \%$ males and $8.3 \%$ females (Table 2,g). Unilateral fetal type posterior cerebral artery and hypoplasia or absence of both a precommunicating segment of the posterior cerebral artery and the posterior communicating artery of the other side were detected in $3.3 \%$ males and $1.7 \%$ females (Table 2,h Figs. 6,10). Bilateral embryonic (fetal) type posterior cerebral arteries with hypoplasia or absence of both precommunicating segments of the posterior cerebral arteries were detected in $3.3 \%$ males and $1.7 \%$ females (Table 2,I Fig.7). Bilateral fetal type posterior cerebral arteries with hypoplasia or absence of one precommunicating segment of a posterior cerebral artery were not noticed in both sexes (Table 2,j).No statistically significant difference was detected in the variations of the posterior part in both sexes.

\section{3-Anatomical variations in the whole circle of Willis (Table3)}

Along with the whole subjects, $45 \%$ demonstrated complete circle of Willis. A complete circle was encountered more in females $(50 \%)$ than males $(40 \%)$. Males exhibited a slightly higher percentage of partially complete circles $(41.7 \%)$ and incomplete circles $(18.3 \%)$ than females (35\% and $15 \%$ respectively); however, these differences were not statistically significant.

\section{4-Sex-related differences in the vessel diameters (Table4)}

Mean diameters of the male vessels was larger than the female vessels. While 
the posterior communicating artery tended to be larger in females (Table 4).

5- Variations in the circle of Willis of the cadavers

Cadavers' examination revealed six cases with complete circle with single anterior communicating and bilateral posterior communicating arteries (Fig.11). In addition, 3 cases of unilateral fetal posterior communicating artery were found (Fig.12). One case of absent posterior communicating artery was detected but the circle was injured before photography.

Table (1): Prevalence of variants in the anterior part of the circle of Willis

\begin{tabular}{|c|c|c|c|c|c|c|c|c|c|c|}
\hline \multirow{2}{*}{ Group } & \multicolumn{10}{|c|}{ Prevalence of variants } \\
\cline { 2 - 10 } & $\mathrm{a}$ & $\mathrm{b}$ & $\mathrm{c}$ & $\mathrm{d}$ & $\mathrm{e}$ & $\mathrm{f}$ & $\mathrm{g}$ & $\mathrm{h}$ & $\mathrm{i}$ & $\mathrm{j}$ \\
\hline Male (n=60) & 35 & 7 & 0 & 3 & 2 & 0 & 9 & 4 & 0 & 0 \\
$(58.3 \%)$ & $(11.7 \%)$ & $(0 \%)$ & $(5 \%)$ & $(3.3)$ & $(0 \%)$ & $(15 \%)$ & $(6.7 \%)$ & $(0 \%)$ & $(0 \%)$ \\
\hline Female (n=60) & 33 & 7 & 0 & 2 & 3 & 0 & 12 & 3 & 0 & 0 \\
& $(55 \%)$ & $(11.7 \%)$ & $(0 \%)$ & $(3.3 \%)$ & $(5 \%)$ & $(0 \%)$ & $(20 \%)$ & $(5 \%)$ & $(0 \%)$ & $(0 \%)$ \\
\hline
\end{tabular}

Note:. The anterior circle variants (a-j) refer to those shown in figure (2).

Table (2): Prevalence of variants in the posterior part of the circle of Willis

\begin{tabular}{|c|c|c|c|c|c|c|c|c|c|c|}
\hline \multirow{2}{*}{ Group } & \multicolumn{9}{|c|}{ Prevalence of variants } & \multicolumn{1}{|c|}{} \\
\cline { 2 - 10 } & $\mathrm{a}$ & $\mathrm{b}$ & $\mathrm{c}$ & $\mathrm{d}$ & $\mathrm{e}$ & $\mathrm{f}$ & $\mathrm{g}$ & $\mathrm{h}$ & $\mathrm{i}$ & $\mathrm{j}$ \\
\hline $\begin{array}{c}\text { Male } \\
\mathrm{n}=60)\end{array}$ & $\begin{array}{c}18 \\
(30 \%)\end{array}$ & $\begin{array}{c}10 \\
(16.7 \%)\end{array}$ & $\begin{array}{c}1 \\
(1.7 \%)\end{array}$ & $\begin{array}{c}13 \\
(21.7 \%)\end{array}$ & $\begin{array}{c}8 \\
(13.3 \%)\end{array}$ & $\begin{array}{c}2 \\
(3.3 \%)\end{array}$ & $\begin{array}{c}4 \\
(6.7 \%)\end{array}$ & $\begin{array}{c}2 \\
(3.3 \%)\end{array}$ & $\begin{array}{c}2 \\
(3.3 \%)\end{array}$ & $\begin{array}{c}0 \\
(0 \%)\end{array}$ \\
\hline $\begin{array}{c}\text { Femal } \\
\mathrm{e}(\mathrm{n}=60)\end{array}$ & $\begin{array}{c}16 \\
(26.7 \\
\%\end{array}$ & $\begin{array}{c}12 \\
(20 \%)\end{array}$ & $\begin{array}{c}5 \\
(8.3 \%)\end{array}$ & $\begin{array}{c}12 \\
(20 \%)\end{array}$ & $\begin{array}{c}7 \\
(11.7 \%)\end{array}$ & $\begin{array}{c}1 \\
(1.7 \%)\end{array}$ & $\begin{array}{c}5 \\
(8.3 \%)\end{array}$ & $\begin{array}{c}1 \\
(1.7 \%)\end{array}$ & $\begin{array}{c}1 \\
(1.7 \%)\end{array}$ & $\begin{array}{c}0 \\
(0 \%)\end{array}$ \\
\hline
\end{tabular}

Note: The posterior circle variants (a-j) refer to those shown in figure (3).

Table (3): Prevalence of the type of the circle of configuration as a whole, complete, partially complete or incomplete

\begin{tabular}{|l|c|c|c|}
\hline \multicolumn{1}{|c|}{ Group } & Entirely complete & Partially complete & Incomplete \\
\hline Male $(\mathrm{n}=60)$ & $24(40 \%)$ & $25(41.7 \%)$ & $11(18.3 \%)$ \\
\hline Female $(\mathrm{n}=60)$ & $30(50 \%)$ & $21(35 \%)$ & $9(15 \%)$ \\
\hline $\begin{array}{l}\text { All subjects } \\
(\mathrm{n}=120)\end{array}$ & $54(45 \%)$ & $46(38.3 \%)$ & $20(16.7 \%)$ \\
\hline
\end{tabular}


Table (4): Mean vessel diameters according to sex

\begin{tabular}{|l|c|c|}
\hline \multirow{2}{*}{ Vessel } & \multicolumn{2}{|c|}{ Vessel diameter $(\mathrm{mm}) \pm$ SD } \\
\cline { 2 - 3 } & & Male \\
\hline P1(The precommunicating segment & & \\
of post. cerebral a) & $1.9 \pm 0.06$ & $1.7 \pm 0.05$ \\
Right & $1.9 \pm 0.07$ & $1.7 \pm 0.04$ \\
Left & $1.9 \pm 0.06$ & $1.7 \pm 0.04$ \\
Both right and left & & \\
\hline P2 (The postcommunicating & $1.9 \pm 0.03$ & $1.8 \pm 0.03$ \\
segment of post. cerebral a) & $1.9 \pm 0.04$ & $1.8 \pm 0.04$ \\
Right & $1.9 \pm 0.03$ & $1.8 \pm 0.03$ \\
Left & & \\
Both right and left & $1.1 \pm 0.01$ & $1.2 \pm 0.02$ \\
\hline PCo (posterior communicating & $1.1 \pm 0.01$ & $1.2 \pm 0.01$ \\
artery) & $1.1 \pm 0.01$ & $1.2 \pm 0.01$ \\
Right & & \\
Left & $4.0 \pm 0.08$ & $3.8 \pm 0.07$ \\
Both right and left & $3.8 \pm 0.06$ & $3.7 \pm 0.06$ \\
\hline ICA (internal carotid artery) & $3.9 \pm 0.07$ & $3.8 \pm 0.06$ \\
Right & & \\
Left & $1.9 \pm 0.04$ & $1.8 \pm 0.05$ \\
Both right and left & $1.8 \pm 0.05$ & $1.7 \pm 0.06$ \\
\hline A1(precommunicating segment of & $1.8 \pm 0.04$ & $1.8 \pm 0.06$ \\
ant. cerebral a) & $1.2 \pm 0.02$ & $1.1 \pm 0.02$ \\
Right & & \\
Left & & \\
Both right and left & & \\
\hline ACo (anterior communicating & & \\
artery) & & \\
\hline
\end{tabular}

$\mathrm{P}<0.05$ is significant.

\section{LEGEND}

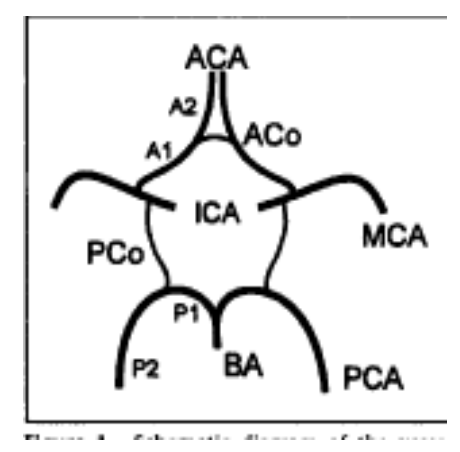

Fig. (1): Schematic diagram of the vessels that form the circle of Willis. The precommunicating segments (A1) of the right and left anterior cerebral arteries (ACA) and an anterior communicating artery (ACO) between them form the anterior part of the circle. The pre-communicating segments (P1) of the right and left posterior cerebral arteries (PCA) form the posterior part of the circle together with the right and left posterior communicating arteries (PCO). The postcommunicating portions of the anterior and posterior cerebral arteries are $\mathrm{A} 2$ and $\mathrm{P} 2$, respectively. $\mathrm{BA}=$ basilar artery, $\mathrm{MCA}=$ middle cerebral artery (after Hartkampe et al., 1998). 


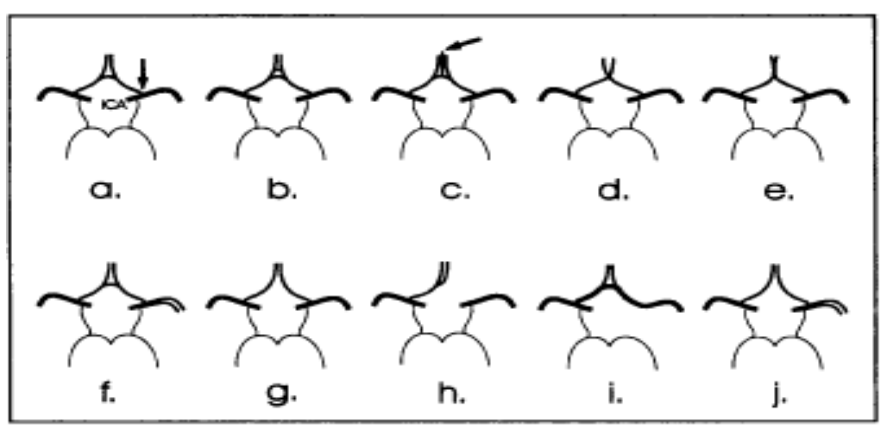

Fig. (2): Schematic diagram of the anatomical variations in the anterior part of the circle of Willis. (a) a single anterior communicating artery. The ICA bifurcates (arrow) into the precommunicating segment of the anterior cerebral artery and the MCA. (b) Two (or more) anterior communicating arteries. (c) Medial artery (arrow) of the corpus callosum arises from the anterior communicating artery. (d) Fusion of the anterior cerebral arteries over a short distance. (e) Anterior cerebral arteries form a common trunk and split distally into two postcommunicating segments. (f) MCA originates from the ICA as two separate trunks. (g) Hypoplasia or absence of an anterior communication. (h) One precommunicating segment of an anterior cerebral artery is hypoplastic or absent, the other precommunicating segment gives rise to both postcommunicating segments of the anterior cerebral arteries. (i) Hypoplasia or absence of an ICA. The contralateral precommunicating segment of the anterior cerebral artery gives rise to both postcommunicating segments. (j) Hypoplasia or absence of an anterior communicating artery and the MCA arises as two separate trunks (after Hartkampe et al., 1998).

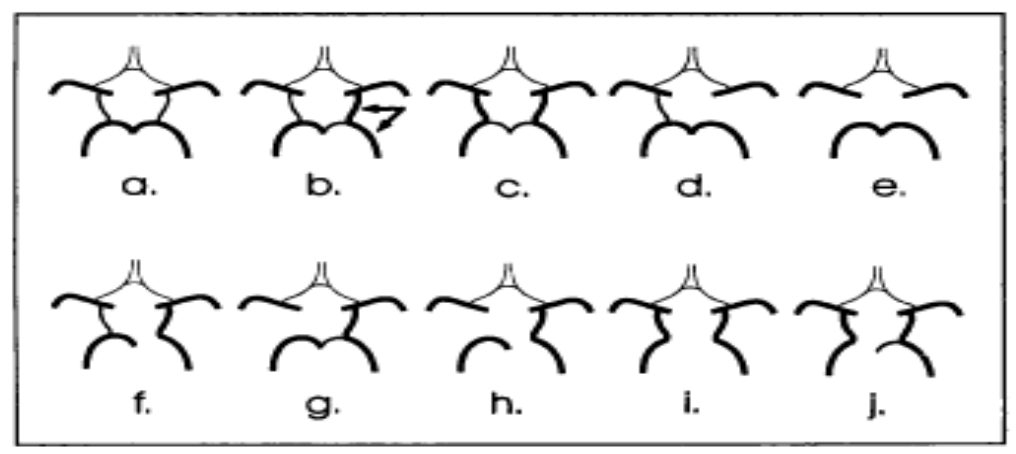

Fig. (3): Schematic diagram of the anatomical variations in the posterior part of the circle of Willis. (a) Bilateral posterior communicating arteries present. (b) Posterior cerebral artery originates predominantly from the ICA. This variant is known as a unilateral embryonic (fetal) type posterior cerebral artery (arrows). (c) Bilateral embryonic type posterior cerebral arteries. (d) Absence of one posterior communicating artery. (e) Hypoplasia or absence of both posterior communicating arteries. (f) Unilateral fetal type posterior cerebral artery and hypoplasia or absence of the precommunicating segment of the posterior cerebral artery. (g) Unilateral fetal type posterior cerebral artery and hypoplasia or absence of the contralateral posterior communicating artery. (h) Unilateral fetal type posterior cerebral artery and hypoplasia or absence of both the precommunicating segment of the posterior cerebral artery and the posterior communicating artery. (i) Bilateral fetal type posterior cerebral arteries with hypoplasia or absence of both precommunicating segments of the posterior cerebral arteries. (j) Bilateral fetal type posterior cerebral arteries with hypoplasia or absence of one precommunicating segment of a posterior cerebral artery (after Hartkampe et al., 1998). 


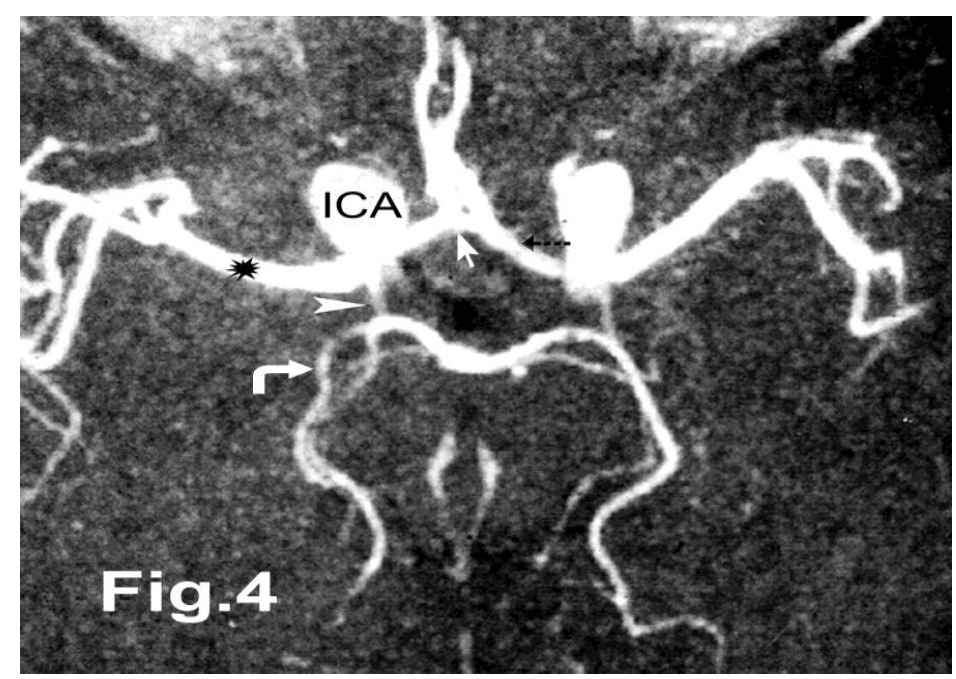

Fig. (4): Magnetic resonance (MR) angiogram of complete circle of Willis in 40-years old male showing internal carotid a (ICA) dividing into the ACA (dotted arrow) and the MCA (*). Notice the single ACO (arrow), the two PCO (arrow head) and the PCA (curved arrow).

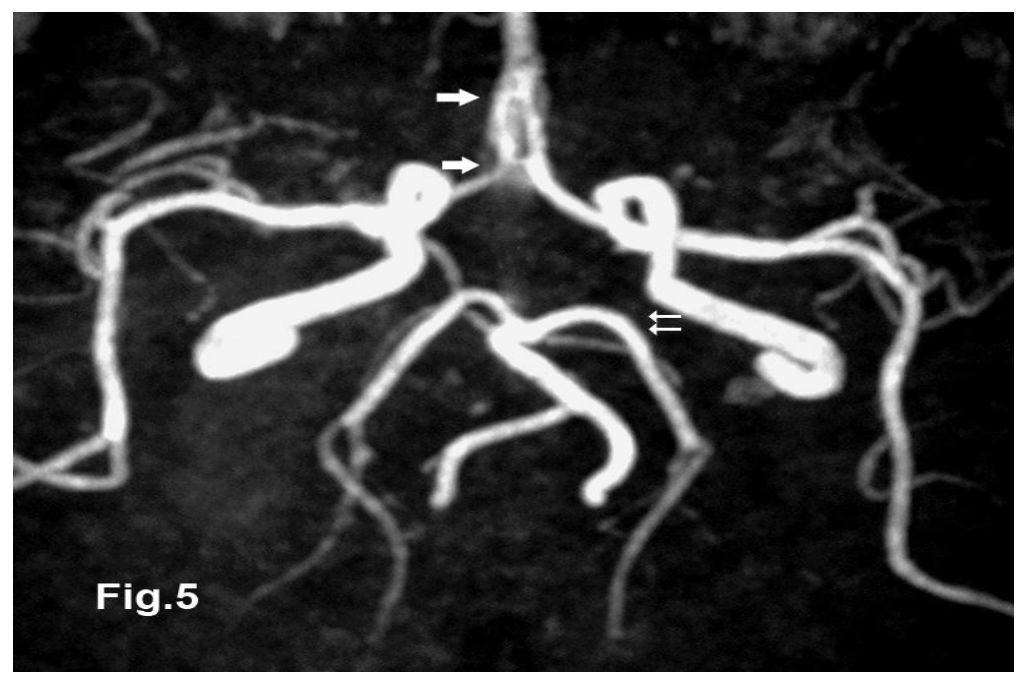

Fig.(5): MR angiogram of a circle of Willis in 42-years old female showing double anterior communicating arteries (arrows). Notice the absent posterior communicating artery (double arrows). 


\section{Kawther A. Hafez et al}

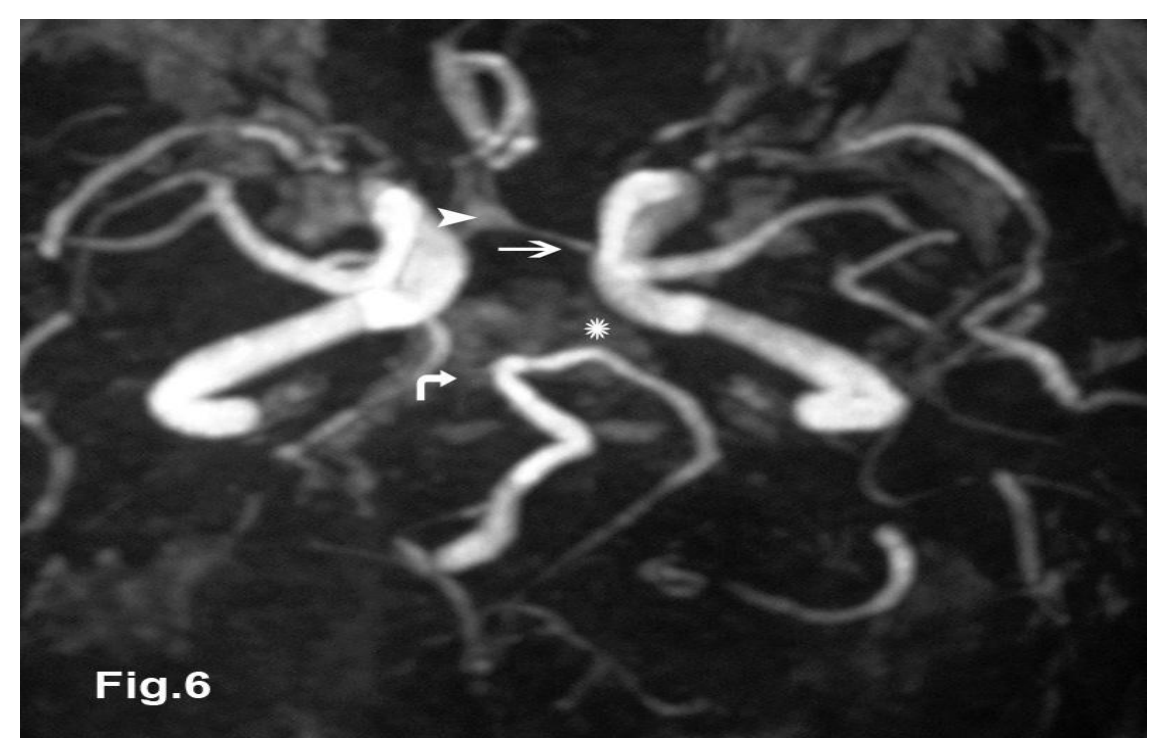

Fig. (6): MR angiogram of a circle of Willis in 76-years old female showing fused anterior communicating arteries forming short trunk (arrowhead) and the two ant. cerebral arteries arising from it. Notice the hypoplastic precommunicating part of the ant. cerebral artery (arrow), absent posterior communicating artery $(*)$ and the fetal posterior communicating artery of the other side with the hypoplastic precommunicating part of the posterior cerebral artery (P1) (curved arrow).

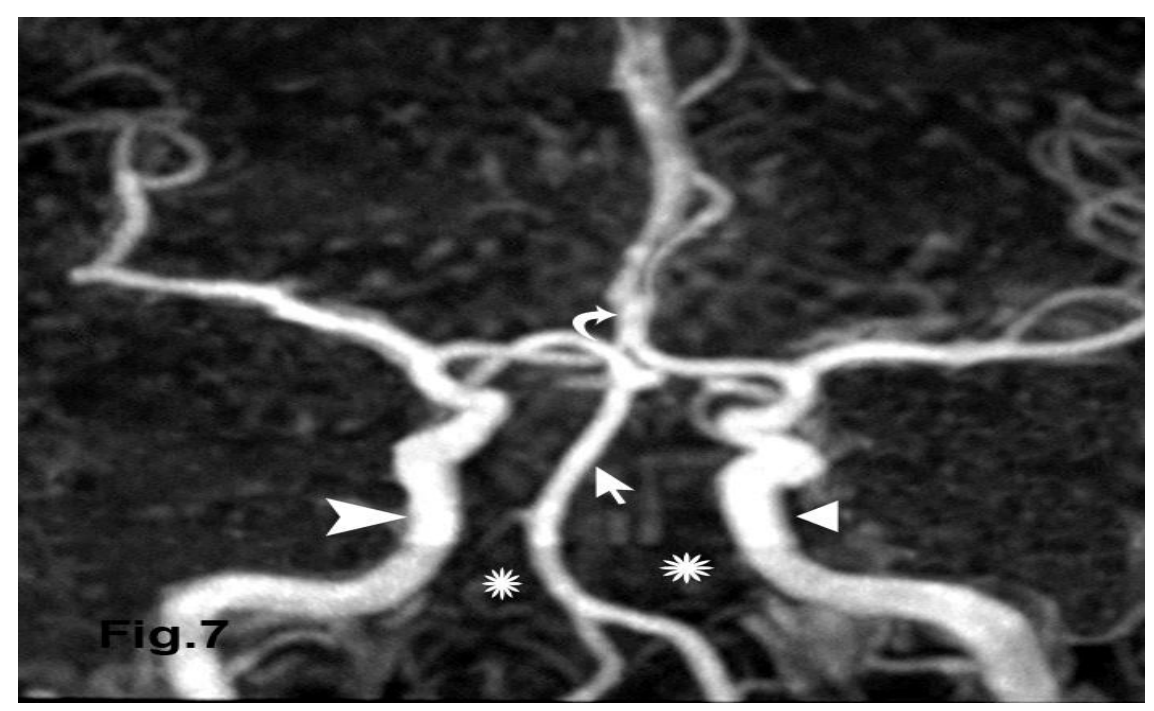

Fig. (7): MR angiogram of a circle of Willis in 52-years old male showing the anterior cerebral arteries forming a common trunk and split distally into two postcommunicating segments (curved arrow). Notice the bilateral fetal cerebral arteries (arrowheads) with the bilateral absent P1 (*) and the basilar artery ascends upward and bifurcates into two divisions (arrow). 


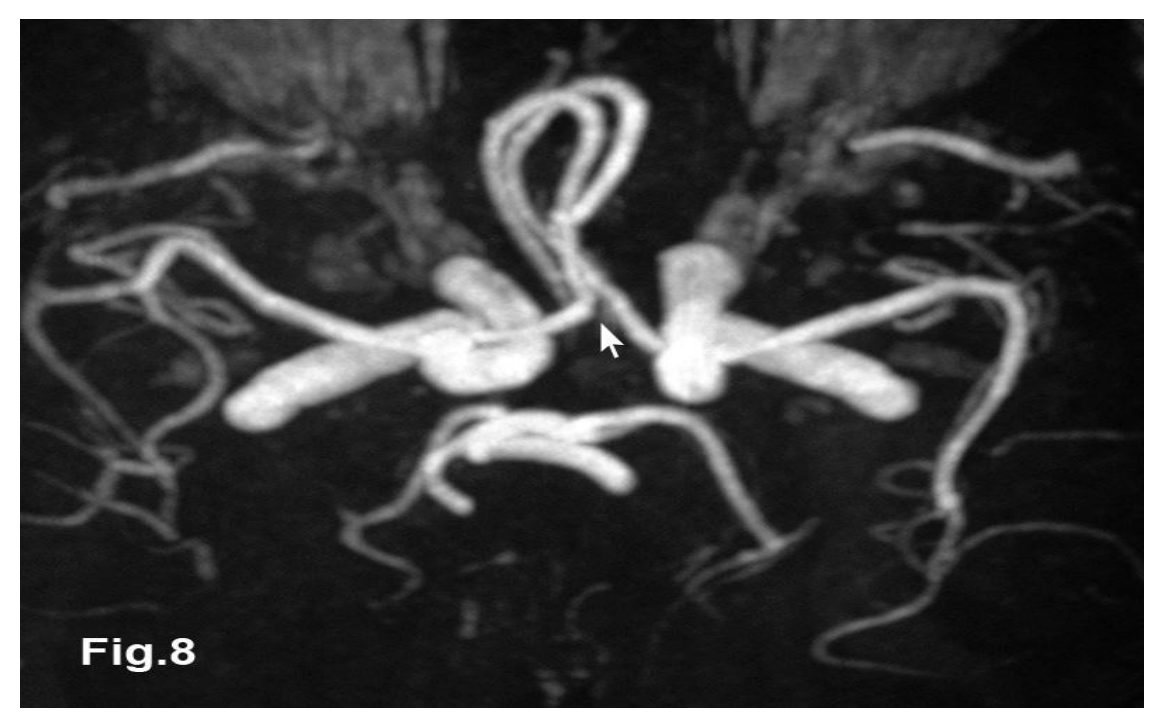

Fig. (8): MR angiogram of a circle of Willis in 53-years old male showing absent anterior communicating artery.

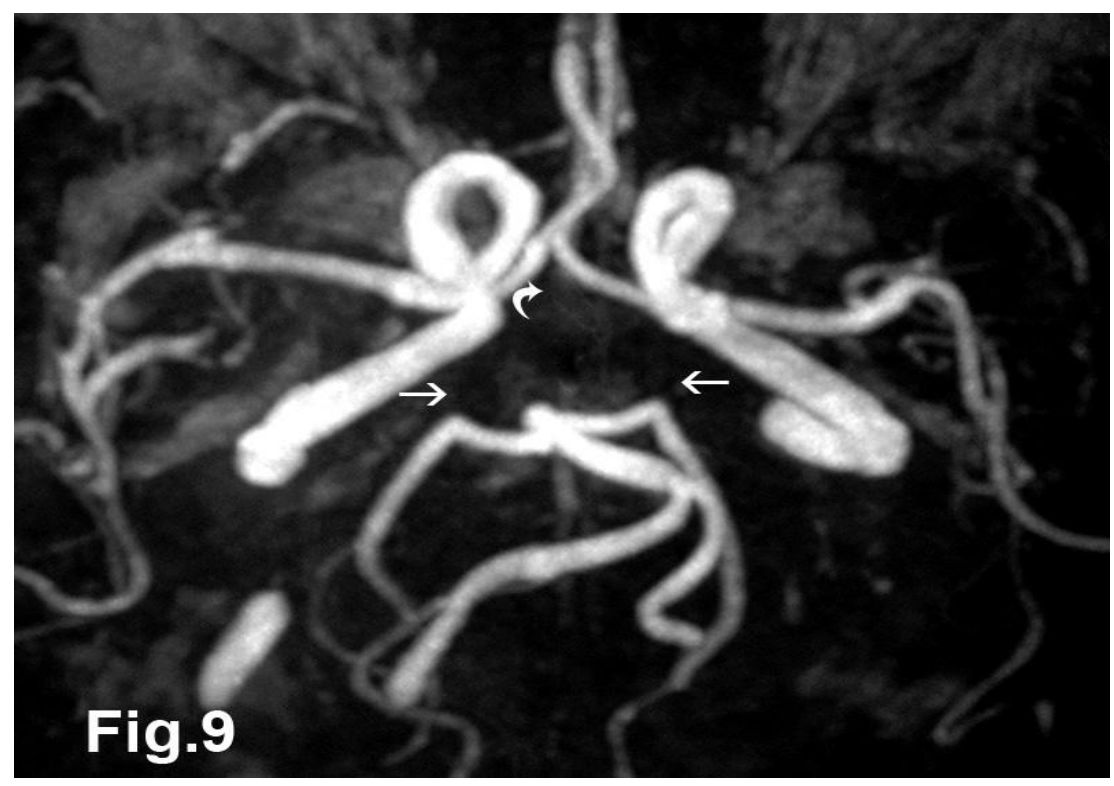

Fig (9): MR angiogram of a circle of Willis in 76-years old male showing absent anterior cerebral artery (curved arrow). Notice the bilateral absent posterior communicating arteries (arrows). 


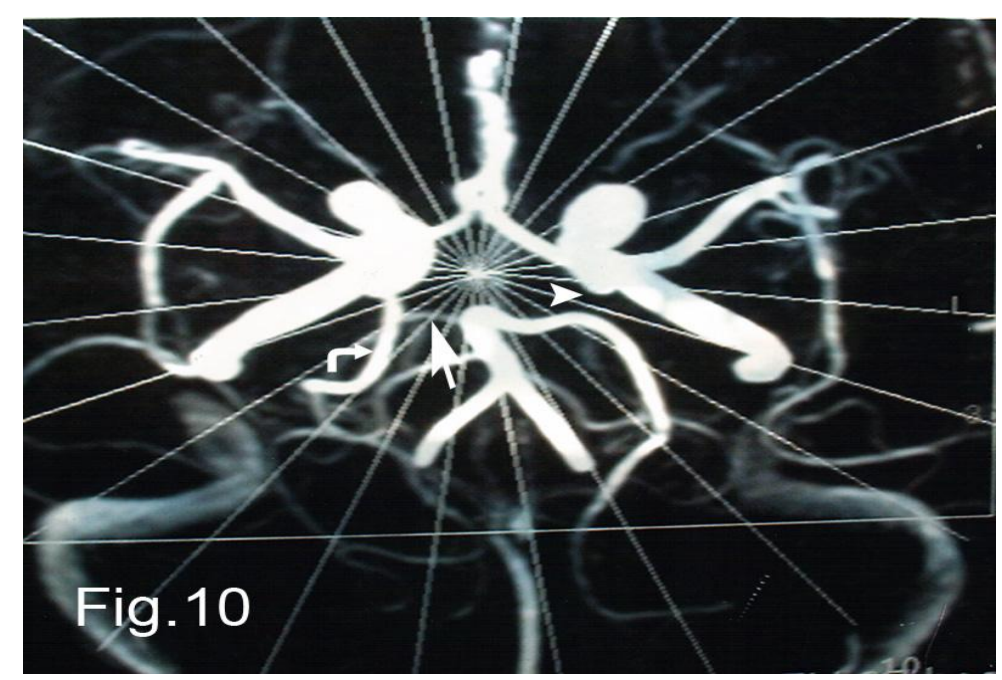

Fig. (10): MR angiogram of a circle of Willis in 40-years old female showing absent posterior communicating artery (arrowhead). Notice the fetal posterior cerebral artery (curved arrow) with the hypoplastic P1(arrow).

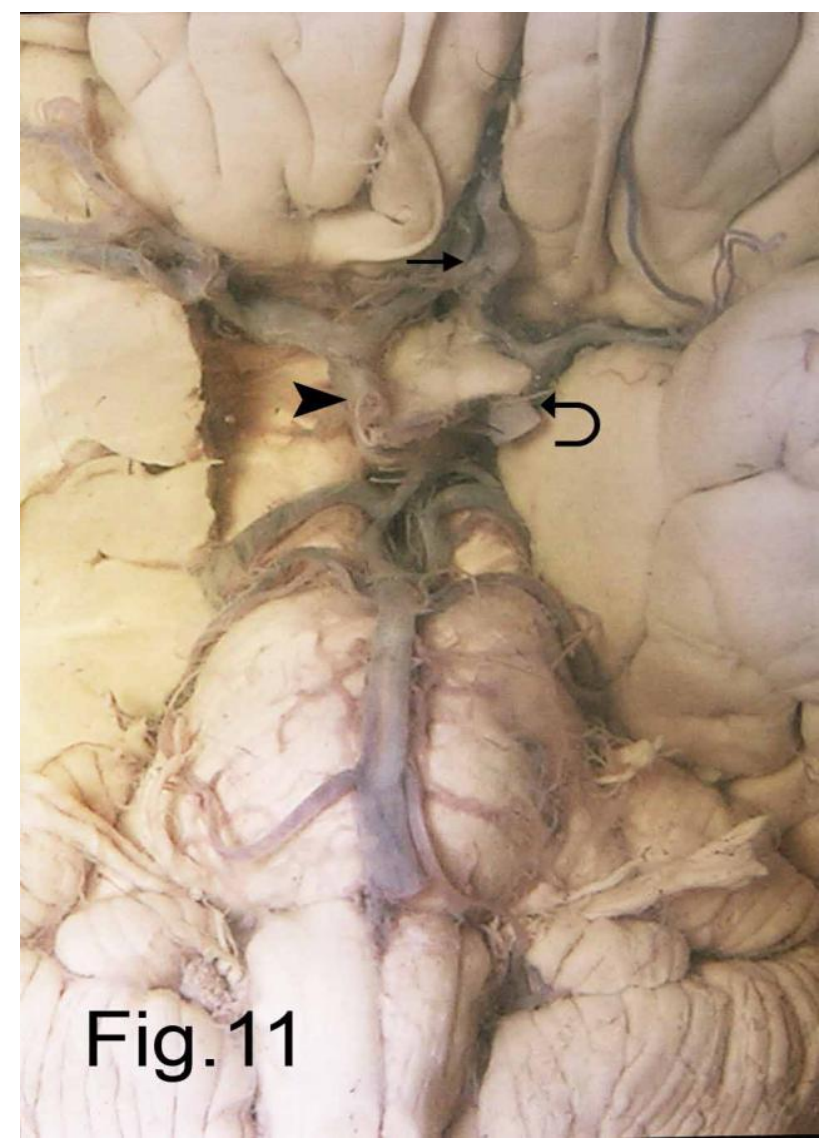

Fig. (11): A photograph of a cadaveric brain showing circle of Willis. The circle is complete with single anterior communicating artery (arrow) and the posterior communicating artery. Note the internal carotid (arrowhead). 


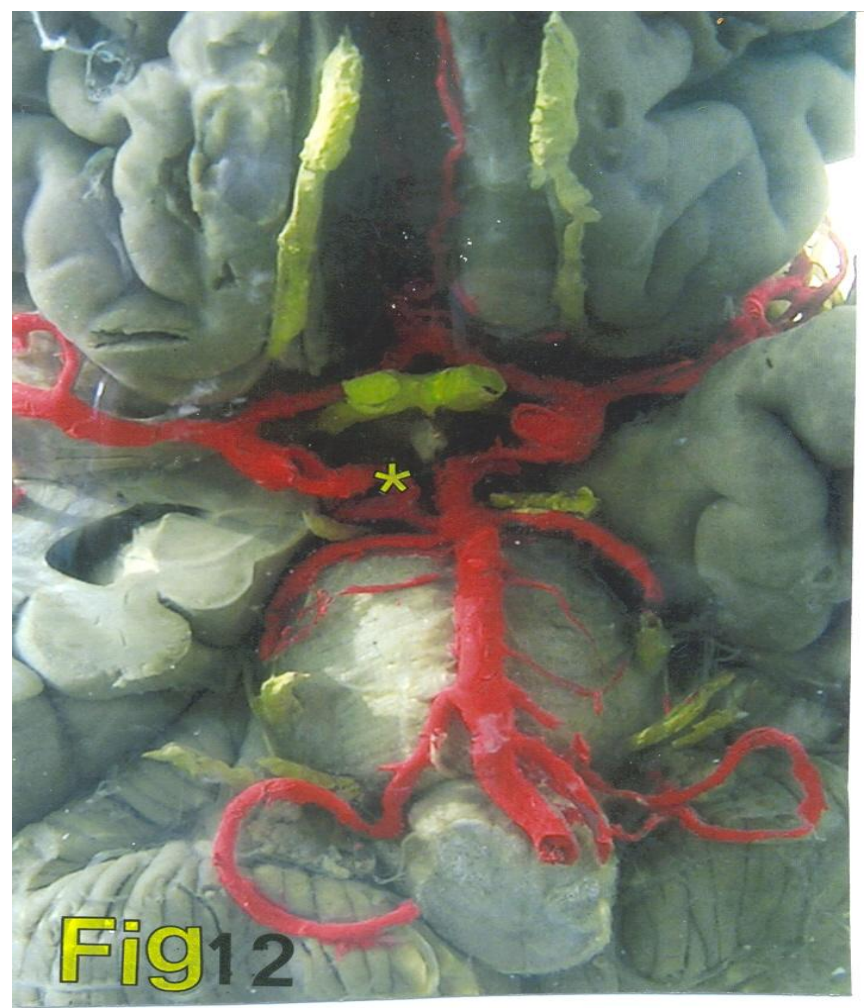

Fig. (12): A photograph of a cadaveric brain showing circle of Willis after red latex injection (from the anatomy museum). The circle has right fetal posterior communicating $\operatorname{artery}(\uparrow)$.

\section{Discussion}

Basic knowledge of the hemodynamic integrity of the circle of Willis is important as its collaterals influence the clinical outcome of patients with internal carotid artery occlusion (Capua et al,. 2000 and Hoksbergen et al., 2003 ). In the present work, anatomical variations of the circle of Willis and the correlation between sex and these variations were studied.

\section{1-Anatomical variations in the anterior part of the circle of Willis}

In this study, the commonest variation in the anterior part of the circle of Willis was the presence of complete arteries with single anterior communicating artery. It was $58.3 \%$ in males and $55 \%$ in females. This was nearly in agreement with Horikoshi et al. (2002) who detected a higher percentage of complete circles $(66.8 \%$ in males and $57 \%$ females) in control patients.
The next common variation in the present study was hypoplasia or absence of the anterior communicating artery; it was $15 \%$ in males and $20 \%$ in females. Similar result was reported by prior studies (Hartkamp et al., 1998, Hartkamp et al., 1999 and Merkkola et al., 2005). In contrary Eftekhar et al. (2006) found only one case in their study to 102 brains.

Two anterior communicating arteries were detected in $11.7 \%$ in males and females. Macchi et al. (1996) accounted a slight lower result $(9 \%)$ in their MRA study on 100 healthy subjects. Other variations in the anterior part were nearly similar to the Hartkamp et al. (1998) and Hartkamp et al. (1999) studies.

In the current work, males were more likely to have variations in the anterior part of the circle than females, though this was not statistically significant. This was in 
accordance with Horikoshi et al. (2002) who concluded that there was a slight higher tendency in males to have variations in the anterior part of the circle than females.

\section{2-Anatomical variations in the posterior part of the circle of Willis}

In the present study, the commonest variation of the posterior part of the circle was the presence of bilateral posterior communicating arteries. It was found in $30 \%$ males and $26.6 \%$ females followed by unilateral posterior communicating artery that was detected in males $(21.7 \%)$ and females (20\%.) Hartkamp et al. (1998) reported comparable results. Fetal type posterior cerebral artery was detected in $16.7 \%$ males and $20 \%$ females. This was nearly in agreement with Eftekhar et al. (2006) and Hoksbergen et al. (2003) who assumed that fetal origin of the posterior cerebral artery was the commonest variation after the ordinary circles. In the current work, bilateral fetal type posterior cerebral arteries were found in $1.7 \%$ males and $8.3 \%$ females. Overbeeke et al. (1991) described in their study that the circle of Willis was fully formed at 7 weeks of gestational age and they classified its configuration into, the transitional pattern which was noticed in early gestational age (in which the diameter of the posterior communicating artery (PCO), equal to the diameter of the posterior cerebral artery PCA), with development of the fetus; this form would be changed into either the adult form (in which the diameter of PCO is lesser than the size of the PCA) or to the fetal form (in which the size of the PCO is wider than that of the precommunicating part (P1) of PCA). Vasovic et al. (2002) proved that in the fetal form, the posterior communicating part of the posterior cerebral artery (P2) originated from the internal carotid artery, by showing that the sympathetic fibers around P2 were continuous with those from the internal carotid artery and not with the fibers around the basilar artery.

Many studies tried to explain the occurrence of the fetal type of the posterior cerebral artery.Former study by Milenkovic et al. (1985) denoted that at the age of $22^{\text {nd }}-$ $24^{\text {th }}$ week of gestational age there were rapid growth of the occipital lobes that demanded an increase in the vasculature of these lobes and that the afferent arteries enlarged to satisfy that demand so the adult form or the fetal form might developed from the transitional form. They added that the preference for an increase in the size of either one of P1 or PCO depended on the most favorable hem-odynamic situation. Other study by Vasovic et al. (2002) postulated that genetic factors were probably responsible for the fetal characteristics of the posterior cerebral artery.

Other variations in the posterior part of the circle were nearly similar to the former studies (Macchi et al., 1996, Hartkamp et al., 1999 and Horikoshi et al., 2002). In the current work, no statistically significant difference were detected in variations in the posterior part in both sexes. On the contrary, Horikoshi et al. (2002) indicated a statistically significant tendency of the females to have variations in the posterior part of the circle.

\section{3-Anatomical variations of the whole circle of Willis}

This work showed that $45 \%$ of the whole subjects demonstrated a complete circle of Willis. Complete circles were encountered more in females $(50 \%)$ than in males (40\%). Horikoshi et al, (2002) detected that the occurrence of complete circles was slightly higher in females than in males.

This study revealed that males exhibited a slight higher percentage of partially complete circles $(41.7 \%)$ and incomplete circles $(18.3 \%)$ than females (35\% and $15 \%$ respectively) however, these differences were not statistically significant. These results were parallel with other researchers (Macchi et al., 1996, and Hartkamp et al., 1998 ) who found no statistically significant difference in the frequency of variation between both sexes.

\section{4-Sex-related differences in the vessel diameters \\ In the current work, the mean} diameters of the male vessels were larger than the female vessels. While the posterior 
communicating artery tended to be larger in the females. These results completely coincided with Hartkamp et al. (1999) and Horikoshi et al. (2002).

\section{5-Variations in the circle of Willis of the cadavers}

Cadavers' examination revealed six cases with complete circle. In addition, 3 cases of unilateral fetal posterior communicating artery and one case of hypoplastic posterior communicating artery were detected. This result compared well with Eftekhar et al. (2006) who studied 102 brains and reported that more than $58 \%$ of them had complete circles and they recorded that $11 \%$ had hypoplastic posterior communicating artery and $27 \%$ of cases demonstrated fetal posterior cerebral arteries.

In conclusion, the present study showed the amazing great variability of the anatomy of the circle of Willis in asymptomatic persons. Nevertheless, there were no marked differences between both sexes in most of the components and the mean diameters of the circle. Therefore, these anatomical variations have to be considered during radiological interpretation and would be reported in the current anatomy text to be aware of all these normal variations.

\section{Acknowledgment}

The authors thank Prof. Dr Yasser Abd El Azeem Professor doctor of Radiology, Ain Shams University and Dr Osama Abd El Gawad, Head of department of Neurosurgery, Kobry El Kobba Military Hospital for their valuable help all through this work.

\section{References}

1. Capua B, Tassi R, Mencattini G. and Passali D. (2000): Non functioning posterior cerebral arteries of circle of Willis in idiopathic sudden hearing loss. The Lancet, 356: 1237- 1239.

2. Eftekhar B, Dadmehr M, Ansari S, Ghodsi M, Nazparvar B. and Ketabchi
E. (2006): Are distribution of variants of the circle of Willis different in different population?- results of an anatomical study and review of literature. Bio.Med. Central Neurol ., 6: 22-32.

3. Hartkamp M. Grand J, Groot J. and Algra A. (1998): Circle of Willis, morphological variation on threedimensional time-of-flight MR angiograms. Radiology; 207: 103 - 111.

4. Hartkamp M, Grond J, Everdingen K, Hillen B. and Mali W. (1999): Circle of Willis collateral flow investigated by magnetic resonance angiography. Stroke., 30:2671 - 2682.

5. Hederson $R$, Eliasziw $M$, Fox A, Rothwell P. and Bamett H.,(2000): Angiographically defined collateral circulation and risk of stroke in patient with severe carotid artery stenosis. Stroke ; 31: 128132.

6. Hoksbergen A, Legemate D, Csiba L, Casati G. and Fulesdi P. (2003): Absent collateral function of the circle of Willis as risk factor for Ischemic stroke. Cerebrovascular diseases.,16: 191- 198.

7. Hoksbergen A., Majoie C., Hulsmans F. and Legemate D. (2003): Assessment of the collateral function of the circle of Willis on three dimensional time- of flight angiography compared with transcranial color code duplex sonography. Am J neuroradiol., 24: 456-462.

8. Horikoshi T, Kiyama I, Amagata Z, Sugita M, and Nukui H. (2002): Magnetic resonance angiographic evidence of sex linked variations in the circle of Willis and the occurrence of cerebral aneurysms. J Neurosurg., 96:697-703.

9. Macchi C, CatiniC., Federico C., Gulisiano M, Pacini P, Cecchi F, Corcos L. and Brizzi E. (1996): Magnetic resonance angiographic evaluation of the circulus arteriosus cerebri(circle of Willis) Ital. J. Anat. Embryol., 101: 115- 123.

10. Merkkola P, Tulla H, Ronkainen A, Soppi V, Oskala A., Koivisto T. and Hippelainen M. (2006): Incomplete circle of Willis and right axillary artery perfusion. Ann Thorac Surg 82: 74-79.

11. Milenkovic Z, Vucetic R. and Puzic $M$. (1985) Asymmetry and anomalies of the circle of Willis in fetal brain. Microsurgical study and functional remarks. Surg Neurol., 24:563-570.

12. Miralles M, Dolz JL, Cotillas J. (1995): The role of the circle of Willis in carotid occlusion: assessment with phase contrast 
MR angiography and transcranial duplex. Eu. J. Vasc. Endovasc. Surg., 10: 424 430.

13. Overbeeke J, Hillen B. and Tulleken C. (1991): A comparative study of the circle of Willis in fetal and adult life. The configuration of the posterior bifurcation of the posterior cerebral artery. J. Anat. 176.,45-54.

14. Ray C, Spalding S, Cothren C, Wang W, Moore E. and Johnson S. (2007): Non invasive imaging and management of neurovascular trauma. World j. Emergency Surgery., 2: 1-31.

15. Schomer D, Marks M, Steinberg G, Johnstone I, Boothroyd D, Ross M., Pelc N. and Enzmann D. (1994): The anatomy of the posterior communicating artery as a risk factor for ischemic cerebral infarction. New England J. M., 330: 1565- 1570.

16. Stock K, Kirsch W, Bongartz G., Steinbrich W. and Radue E. (1996): Anatomic evaluation of the circle of Willis: MR angiography versus intraarterial digital subtraction angiography. Am. J. Neuroradiol: 17 1495- 1499.

17. Vasovic L., Milenkovic Z. and Pavlovic S. (2002): Comparative morphological variations and abnormalities of circles of Willis: a minireview including two personal cases. Neurosurg Rev.; 25: 247-251.

18. William P, Bannister L, Berry M, Dyson $M$, Dussek JE and Ferguson $M$ W. (1995): Gray's Anatomy. 38 ${ }^{\text {th }}$ edition., Churchill Livingstone. P: 1529. 


\title{
الاختلافات التشريحية في حلقة ويليس في الأكور و الاناث باستخدام الرنين

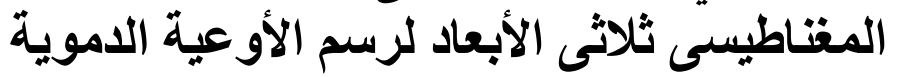

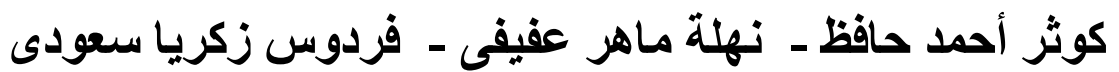

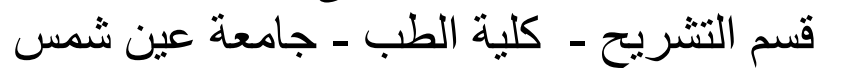

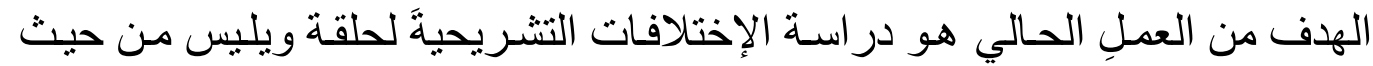

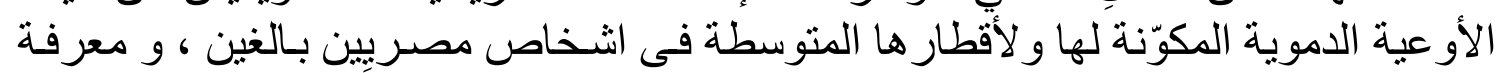

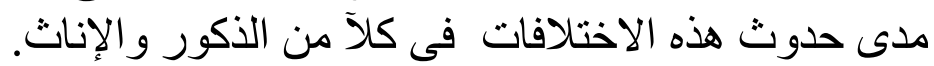

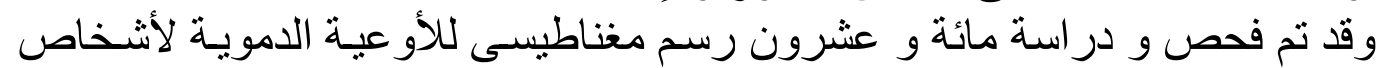

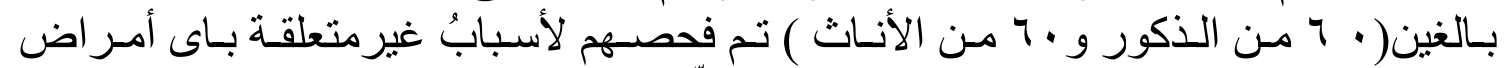

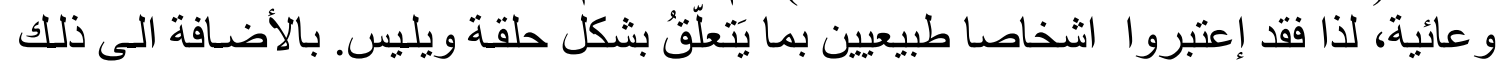

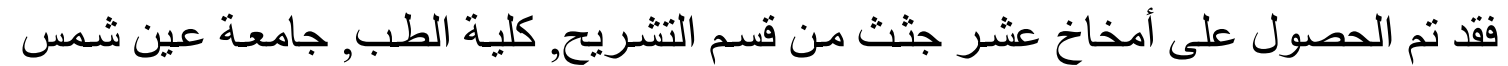

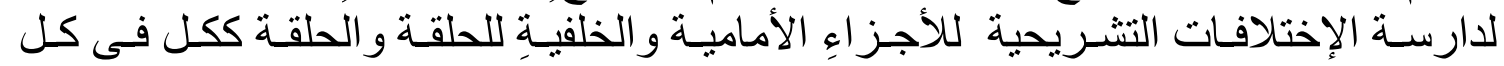

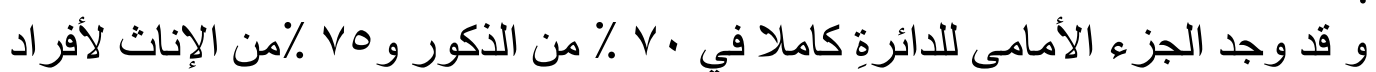

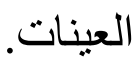

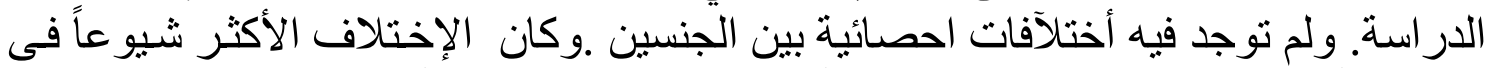

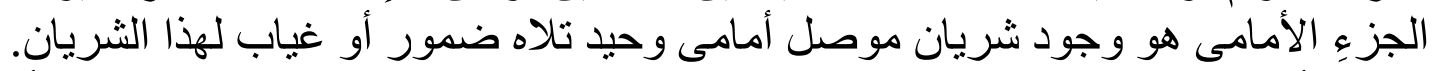

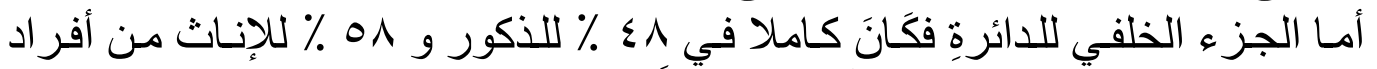

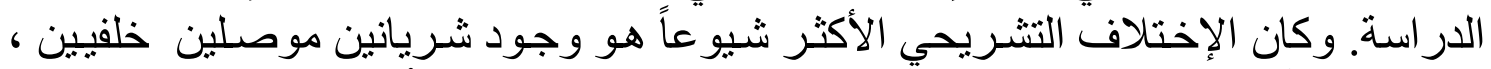

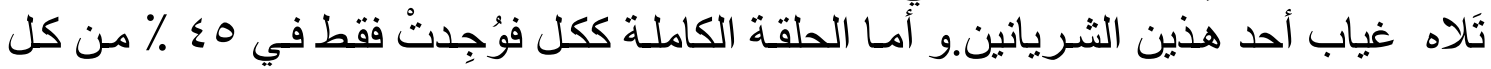

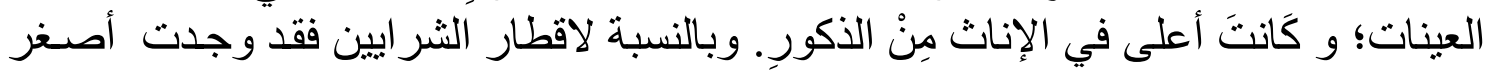

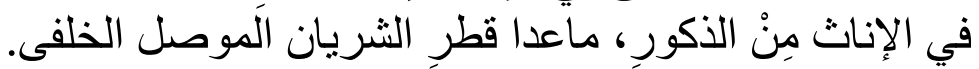

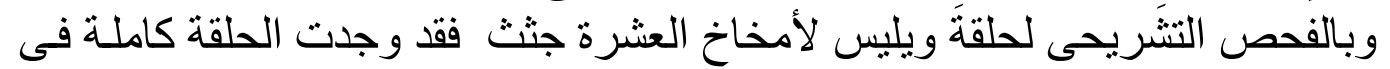

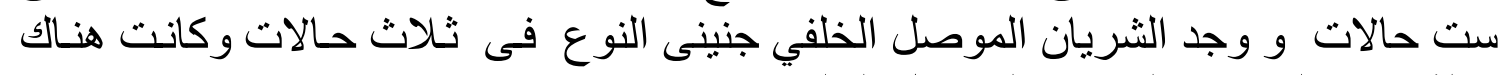

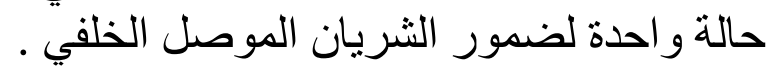

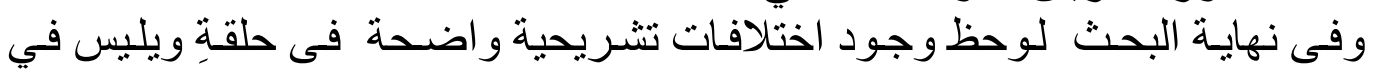

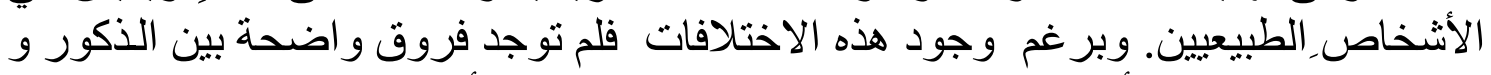

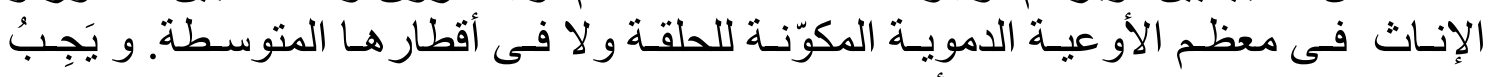

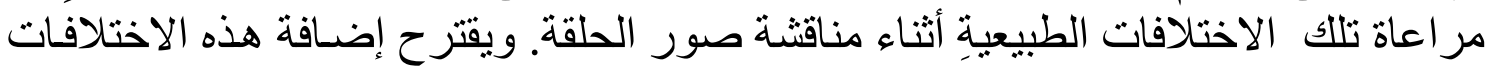
الطبيعيةِ فى كتب التشريح للإلمام بها. 